\author{
for \\ the \\ communication \\ entitled
}

\title{
Copper Sulfate-Pentahydrate-1,10-Phenanthroline Catalyzed Amidations of Alkynyl Bromides. Synthesis of Heteroaromatic Amine Substituted Ynamides.
}

\author{
authored by \\ Yanshi Zhang, Richard P. Hsung*, Michael R. Tracey, Kimberly C. M. Kurtz, and Eymi L. Vera
}

Department of Chemistry, University of Minnesota, Minneapolis, MN 55455 


\section{A Representative Procedure for the Coupling Reaction: Synthesis of Ynamide 30.}

To a solution of 1-bromo-2-triisopropylsilylacetylene $(575.0 \mathrm{mg}, 2.20 \mathrm{mmol})$ in $2 \mathrm{~mL}$ of freshly anhydrous toluene in a reaction vial were added $N$-benzyltoluenesulfonamide $(522.0 \mathrm{mg}, 2.00 \mathrm{mmol}), \mathrm{K}_{2} \mathrm{CO}_{3}$ (560.0 mg, $4.00 \mathrm{mmol}), \mathrm{CuSO}_{4}-5 \mathrm{H}_{2} \mathrm{O}(50.0 \mathrm{mg}, 0.20 \mathrm{mmol})$, and 1,10-phenanthroline (74.0 mg, $0.40 \mathrm{mmol}$ ). The reaction mixture was caped under a blanket of nitrogen and heated in an oil bath at $60-65^{\circ} \mathrm{C}$ for $32 \mathrm{~h}$. The progress of the reaction was monitored using TLC analysis. Upon completion, the reaction mixture was cooled to room temperature and diluted with $12 \mathrm{~mL}$ of chloroform. The resulting mixture was filtered through a pipette-size celite column, and the filtrate was concentrated in vacuo. The crude residue was purified using silica gel column flash chromatography [gradient eluent: 0-10\% EtOAc in hexane] to give ynamide 30 (852.0 $\mathrm{mg}, 97 \%$ ) as clear oil.

\section{Notes:}

1. The same reaction at a 0.50 -mmol scale afforded the title compound in comparable yield.

2. The amidation reaction is also suitable for larger scale preparations. We were able to prepare ynamide $\mathbf{1 2}$ at a $6.69-\mathrm{mmol}$ scale and obtained $2.52 \mathrm{~g}$ of $\mathbf{1 2}$ in $70 \%$ yield.

\section{CHARACTERIZATIONS}

Ynamide 8 (39.0 $\mathrm{mg}, 73 \%$ yield).

$\mathrm{R}_{\mathrm{f}}=0.41$ (10\% EtOAc in Hexanes); clear oil; ${ }^{1} \mathrm{H}$ NMR $\left(300 \mathrm{MHz}, \mathrm{CDCl}_{3}\right) \delta 3.85$ (s, $\left.3 \mathrm{H}\right), 4.72(\mathrm{~s}, 2 \mathrm{H}), 7.22-$ 7.45 (m, $10 \mathrm{H}) ;{ }^{13} \mathrm{C}$ NMR $\left(75 \mathrm{MHz}, \mathrm{CDCl}_{3}\right) \delta 54.2,54.3,71.3,83.1,123.2,127.7,128.2,128.3,128.7,131.3$, 136.0, 155.8; IR (thin film) $\mathrm{cm}^{-1} 2954$ (w), 2238 (m), 1729 (s), 1443 (s), 1294 (s), 755 (m); mass spectrum (APCI): $\mathrm{m} / \mathrm{e}$ (\% relative intensity) $266(100)(\mathrm{M}+\mathrm{H})^{+}, 226(25), 195$ (24), 181 (34), 91 (34).

Ynamide 11 (99.0 $\mathrm{mg}, 98 \%$ yield).

$\mathrm{R}_{\mathrm{f}}=0.27(20 \%$ EtOAc in Hexanes $)$; white solid $67-69^{\circ} \mathrm{C} ;(\alpha)_{\mathrm{D}}{ }^{23}=-60.972\left(c=1.44, \mathrm{CH}_{2} \mathrm{Cl}_{2}\right)$;

${ }^{1} \mathrm{H}$ NMR $\left(300 \mathrm{MHz}, \mathrm{CDCl}_{3}\right) \delta 0.94$ (s, $\left.3 \mathrm{H}\right), 1.05$ (s, $\left.3 \mathrm{H}\right), 1.35$ (s, $\left.3 \mathrm{H}\right), 1.63$ (ddd, $1 \mathrm{H}, J=4.3,8.6,12.9 \mathrm{~Hz}$ ), 1.79-1.93 (m , 2 H), $2.04(\mathrm{~m}, 1 \mathrm{H}), 2.40$ (d, $1 \mathrm{H}, J=4.5 \mathrm{~Hz}), 7.24-7.31$ (m, $3 \mathrm{H}), 7.40-7.44(\mathrm{~m}, 2 \mathrm{H}) ;{ }^{13} \mathrm{C} \mathrm{NMR}$ $\left(75 \mathrm{MHz} \mathrm{CDCl}_{3}\right) \delta 12.2,18.1,18.5,23.1,49.5,54.2,74.1,74.3,78.3,123.1,127.5,128.2,131.2,178.0$; IR (thin film) $\mathrm{cm}^{-1} 2968(\mathrm{w}), 2236(\mathrm{~m}), 1732$ (s), 1392 (s); mass spectrum (APCI): m/e (\% relative intensity) 254 (100) $(\mathrm{M}+\mathrm{H})^{+}$.

Ynamide 12 (2.52 g, 70\% yield).

$\mathrm{R}_{\mathrm{f}}=0.34$ (25\% EtOAc in hexanes); yellow oil; $(\alpha)_{\mathrm{D}}{ }^{23}=-39.127\left(c=0.63, \mathrm{CH}_{2} \mathrm{Cl}_{2}\right)$;

${ }^{1} \mathrm{H}$ NMR $\left(300 \mathrm{MHz}, \mathrm{CDCl}_{3}\right): \delta 0.05(\mathrm{~s}, 6 \mathrm{H}), 0.90(\mathrm{~s}, 9 \mathrm{H}), 0.93(\mathrm{~s}, 3 \mathrm{H}), 1.01(\mathrm{~s}, 3 \mathrm{H}), 1.26(\mathrm{~s}, 3 \mathrm{H}), 1.52-1.68$ (m, $5 \mathrm{H}$ ), 1.80 (dddd, $2 \mathrm{H}, J=4.5,11.2,11.2,20.3$ ), 2.02 (ddd, $1 \mathrm{H}, J=4.5,9.2,17.6), 2.38$ (m, $3 \mathrm{H}), 3.63$ (t, 2 
$\mathrm{H}, J=5.1) ;{ }^{13} \mathrm{C} \mathrm{NMR}\left(75 \mathrm{MHz}, \mathrm{CDCl}_{3}\right) \delta-5.4(2 \mathrm{C}), 12.0,18.1,18.2,18.4,18.5,23.1,25.4,25.8(3 \mathrm{C}), 31.9$, 32.6, 49.2, 54.0, 62.6, 68.8, 73.2, 73.6, 178.3. IR (thin film) $\mathrm{cm}^{-1} 2858$ (s), 2257 (m), 1736 (s), 1255 (s), 1107 (s), 828 (s); mass spectrum (ESI): m/e (\% relative intensity) $386.2(100)\left(\mathrm{M}+\mathrm{Na}^{+}\right) ; \mathrm{m} / \mathrm{e}$ calcd for $\mathrm{C}_{21} \mathrm{H}_{37} \mathrm{NO}_{2} \mathrm{SiNa}\left(\mathrm{M}+\mathrm{Na}^{+}\right)$: 386.2486, found: 386.2487 .

Ynamide 13 (74.0 mg, 97\% yield).

$\mathrm{R}_{\mathrm{f}}=0.33\left(20 \%\right.$ EtOAc in Hexanes); $\mathrm{mp} 92-94^{\circ} \mathrm{C} ;(\alpha)_{\mathrm{D}}{ }^{23}=88.563\left(c=3.34, \mathrm{CH}_{2} \mathrm{Cl}_{2}\right)$;

${ }^{1} \mathrm{H}$ NMR (300 MHz, CDCl $) \delta 0.87$ (s, $3 \mathrm{H}$ ), 0.99 (s, $3 \mathrm{H}$ ), 1.03 (s, $\left.3 \mathrm{H}\right), 1.54$ (ddd, $1 \mathrm{H}, J=4.2,9.3,13.5$ ), 1.70-1.84 (m, $2 \mathrm{H}), 1.95$ (dddd, $J=2.1,4.5,9.9,13.5 \mathrm{~Hz}), 3.58$ (d, $J=21 . \mathrm{Hz}), 7.20-7.30$ (m, $3 \mathrm{H}), 7.33-7.42$ $(\mathrm{m}, 2 \mathrm{H}) ;{ }^{13} \mathrm{C}$ NMR $\left(75 \mathrm{MHz}, \mathrm{CDCl}_{3}\right) \delta 9.4,17.8,18.5,26.5,30.3,53.9,71.3,71.9,80.4,123.1,127.5,128.2$, 131.2, 179.4; IR (thin film) $\mathrm{cm}^{-1} 2964$ (w), 2236 (m), 1733 (s), 1400 (s), 1138 (m); mass spectrum (APCI): m/e (\% relative intensity) $254(100)(\mathrm{M}+\mathrm{H})^{+}$.

Ynamide 15 ( $90.0 \mathrm{mg}, 81 \%$ yield).

$\mathrm{R}_{\mathrm{f}}=0.53\left(33 \%\right.$ EtOAc in hexane); $\mathrm{mp} 48-50^{\circ} \mathrm{C} ;(\alpha)_{\mathrm{D}}{ }^{23}=-176.27\left(c=1.02, \mathrm{CH}_{2} \mathrm{Cl}_{2}\right)$;

${ }^{1} \mathrm{H} \mathrm{NMR}\left(300 \mathrm{MHz}, \mathrm{CDCl}_{3}\right) \delta 0.80(\mathrm{~d}, 3 \mathrm{H}, J=6.6 \mathrm{~Hz}), 0.90(\mathrm{~s}, 21 \mathrm{H}), 2.83(\mathrm{~s}, 3 \mathrm{H}), 3.90(\mathrm{dq}, 1 \mathrm{H}, J=6.6,8.7$ $\mathrm{Hz}), 4.97$ (d, $1 \mathrm{H}, J=8.7 \mathrm{~Hz}), 7.18-7.26$ (m, $2 \mathrm{H}), 7.30-7.42(\mathrm{~m}, 3 \mathrm{H}) ;{ }^{13} \mathrm{C} \mathrm{NMR}\left(75 \mathrm{MHz}, \mathrm{CDCl}_{3}\right) \delta 11.3,15.0$, 18.6, 28.9, 55.5, 64.3, 69.6, 95.8, 128.0, 128.5, 134.6, 157.7; IR (thin film) $\mathrm{cm}^{-1} 2941$ (s), 2864 (s), 2171 (s), $1735(\mathrm{~s}), 1460(\mathrm{~m}), 1426(\mathrm{~m}), 1398(\mathrm{~s})$; mass spectrum (EI): m/e (\% relative intensity) $370(3)\left(\mathrm{M}^{+}\right), 327(100)$ $\left(\mathrm{M}^{+}-\mathrm{P} \mathrm{Pr}\right)$.

Ynamide 16 (132.0 mg, 37\% yield).

$\mathrm{R}_{\mathrm{f}}=0.15\left(50 \%\right.$ EtOAc in hexane); $(\alpha)_{\mathrm{D}}^{23}=-2.85\left(c=13.2, \mathrm{CH}_{2} \mathrm{Cl}_{2}\right)$;

${ }^{1} \mathrm{H} \mathrm{NMR}\left(500 \mathrm{MHz}, \mathrm{CDCl}_{3}\right) \delta 0.67(\mathrm{~d}, 3 \mathrm{H}, J=6.5 \mathrm{~Hz}), 2.73(\mathrm{~s}, 3 \mathrm{H}), 3.70(\mathrm{~s}, 3 \mathrm{H}), 3.77(\mathrm{~m}, 1 \mathrm{H}), 4.95(\mathrm{~d}, 1 \mathrm{H}$, $J=8.0 \mathrm{~Hz}), 6.95(\mathrm{~d}, 1 \mathrm{H}, J=8.5 \mathrm{~Hz}), 7.13-7.18(\mathrm{~m}, 2 \mathrm{H}), 7.21-7.22(\mathrm{~m}, 2 \mathrm{H}), 7.24-7.31(\mathrm{~m}, 3 \mathrm{H}), 7.53(\mathrm{~d}, 1 \mathrm{H}$, $J=9.0 \mathrm{~Hz}), 7.55(\mathrm{~d}, 1 \mathrm{H}, J=7.0 \mathrm{~Hz}), 7.64(\mathrm{~d}, 1 \mathrm{H}, J=7.5 \mathrm{~Hz}) ;{ }^{13} \mathrm{C} \mathrm{NMR}\left(125 \mathrm{MHz}, \mathrm{CDCl}_{3}\right) \delta 14.9,29.0,55.6$, 56.8, 64.4, 66.1, 90.7, 104.2, 106.9, 113.4, 123.9, 125.5, 126.8, 127.8, 128.2, 128.5, 128.6, 128.9, 134.3, 135.0, 157.6, 158.1; IR (thin film) $\mathrm{cm}^{-1} 3057$ (w), 2936 (w), 2240 (s), 1727 (s), 1590 (m), 1435 (s), 1395 (s), 1271 (s); mass spectrum (CI): $\mathrm{m} / \mathrm{e}\left(\%\right.$ relative intensity) $371(100)(\mathrm{M}+\mathrm{H})^{+}, 357(5), 191(10)$.

Ynamide 17 (55.0 $\mathrm{mg}, 61 \%$ yield).

$\mathrm{R}_{\mathrm{f}}=0.30$ (33\% EtOAc in Hexanes); $\mathrm{mp} 51-52^{\circ} \mathrm{C} ;(\alpha)_{\mathrm{D}}{ }^{23}=-148.44\left(c=4.24, \mathrm{CH}_{2} \mathrm{Cl}_{2}\right)$;

${ }^{1} \mathrm{H}$ NMR $\left(300 \mathrm{MHz}, \mathrm{CDCl}_{3}\right) \delta 0.77(\mathrm{~d}, 3 \mathrm{H}, J=6.6 \mathrm{~Hz}), 0.83(\mathrm{t}, 3 \mathrm{H}, J=6.9 \mathrm{~Hz}), 1.08-1.28(\mathrm{~m}, 6 \mathrm{H}), 1.28-1.40$ (m, $2 \mathrm{H}), 2.18(\mathrm{t}, 2 \mathrm{H}, J=6.9 \mathrm{~Hz}), 2.83(\mathrm{~s}, 3 \mathrm{H}), 3.88(\mathrm{dq}, 1 \mathrm{H}, J=6.6,8.7 \mathrm{~Hz}), 4.90(\mathrm{~d}, 1 \mathrm{H}, J=8.7 \mathrm{~Hz}), 7.20-$ 7.27 (m, $2 \mathrm{H}), 7.30-7.42(\mathrm{~m}, 3 \mathrm{H}) ;{ }^{13} \mathrm{C} \mathrm{NMR}\left(75 \mathrm{MHz}, \mathrm{CDCl}_{3}\right) \delta 14.1,14.8,18.6,22.5,28.2,28.9,31.3,55.6$, 64.1, 70.9, 72.1, 127.8, 128.3, 128.4, 135.0, 158.6; IR (thin film) $\mathrm{cm}^{-1} 2930(\mathrm{~m}), 2260$ (w), 1729 (s), $1428(\mathrm{~m})$, $1401(\mathrm{~m})$; mass spectrum (APCI): $\mathrm{m} / \mathrm{e}$ (\% relative intensity) $331(100)(\mathrm{M}+\mathrm{MeOH}+\mathrm{H})^{+}, 317\left(\mathrm{M}+\mathrm{H}_{2} \mathrm{O}+\right.$ $\mathrm{H})^{+}, 299(\mathrm{M}+\mathrm{H})^{+}, 191(96)$. 
Ynamide 18 (78.0 mg, 56\% yield).

$\mathrm{R}_{\mathrm{f}}=0.45$ (10\% EtOAc in Hexanes); clear oil; $(\alpha)_{\mathrm{D}}^{23}=162.43\left(c=2.47, \mathrm{CH}_{2} \mathrm{Cl}_{2}\right)$;

${ }^{1} \mathrm{H}$ NMR $\left(300 \mathrm{MHz}, \mathrm{CDCl}_{3}\right) \delta 1.71(\mathrm{~d}, 3 \mathrm{H}, J=7.0 \mathrm{~Hz}), 3.84(\mathrm{~s}, 3 \mathrm{H}), 5.42(\mathrm{q}, 1 \mathrm{H}, J=7.0 \mathrm{~Hz}), 7.26-7.33(\mathrm{~m}, 8$ H), $7.34(\mathrm{~d}, J=6.6 \mathrm{~Hz}) ;{ }^{13} \mathrm{C} \mathrm{NMR}\left(75 \mathrm{MHz}, \mathrm{CDCl}_{3}\right) \delta 18.8,54.2,56.2,73.4,80.6,123.4,127.0,127.6,128.0$, 128.4, 128.6, 131.2, 140.7, 155.6; IR (thin film) $\mathrm{cm}^{-1} 2958$ (w), 2236 (m), 1730 (s), 1440 (m), 1290 (s); mass spectrum (APCI): m/e (\% relative intensity) $280(100)(\mathrm{M}+\mathrm{H})^{+}, 236(23), 195$ (55), 136 (14);.

Ynamide 19 ( $74.0 \mathrm{mg}, 63 \%$ yield).

$\mathrm{R}_{\mathrm{f}}=0.64\left(10 \%\right.$ EtOAc in Hexanes); clear oil; $(\alpha)_{\mathrm{D}}^{23}=50.538\left(c=10.4, \mathrm{CH}_{2} \mathrm{Cl}_{2}\right)$;

${ }^{1} \mathrm{H}$ NMR $\left(300 \mathrm{MHz}, \mathrm{CDCl}_{3}\right) \delta 0.84(\mathrm{brs}, 3 \mathrm{H}), 0.94(\mathrm{~d}, 6 \mathrm{H}, J=6.6 \mathrm{~Hz}), 1.06(\mathrm{~d}, 1 \mathrm{H}, J=12.0 \mathrm{~Hz}), 1.10(\mathrm{~d}, 1$ $\mathrm{H}, J=12.0 \mathrm{~Hz}), 1.41-1.60(\mathrm{~m}, 2 \mathrm{H}), 1.71(\mathrm{~d}, 2 \mathrm{H}, J=11.6 \mathrm{~Hz}), 1.96-2.16(\mathrm{~m}, 1 \mathrm{H}), 2.16(\mathrm{~d}, 1 \mathrm{H}, J=11.6 \mathrm{~Hz})$, 4.61-4.82 (m, $3 \mathrm{H}), 7.22-7.43(\mathrm{~m}, 10 \mathrm{H}) ;{ }^{13} \mathrm{C} \mathrm{NMR}\left(75 \mathrm{MHz}, \mathrm{CDCl}_{3}\right) \delta 16.9,20.8,22.1,23.9,26.6,31.5,34.3$, 41.2, 47.3, 53.7, 71.2, 78.0, 83.7, 123.6, 127.3, 128.0, 128.3, 128.4, 128.6, 130.8, 136.4, 155.0; IR (thin film) $\mathrm{cm}^{-1} 2953(\mathrm{w}), 2243(\mathrm{w}), 1719(\mathrm{~s}), 1397(\mathrm{~m}), 1291(\mathrm{~m}), 1235(\mathrm{~m})$; mass spectrum (APCI): m/e (\% relative intensity) $422(96)(\mathrm{M}+\mathrm{MeOH}+\mathrm{H})^{+}, 390(10)(\mathrm{M}+\mathrm{H})^{+}, 346$ (43), 284 (34), 240 (100), 226 (73), 208 (63), 91 (41).

Ynamide 20 (123.0 mg, 59\% yield).

$\mathrm{R}_{\mathrm{f}}=0.63$ (10\% EtOAc in Hexanes); clear oil; ${ }^{1} \mathrm{H}$ NMR $\left(300 \mathrm{MHz}, \mathrm{CDCl}_{3}\right) \delta 0.89$ (t, $\left.3 \mathrm{H}, J=6.7 \mathrm{~Hz}\right), 1.22-$ $1.42(\mathrm{~m}, 6 \mathrm{H}), 1.73$ (quint, $2 \mathrm{H}, J=7.2 \mathrm{~Hz}$ ), 3.56 (t, $2 \mathrm{H}, J=7.2 \mathrm{~Hz}$ ), $3.82(\mathrm{~s}, 3 \mathrm{H}), 7.21-7.35$ (m, $3 \mathrm{H}), 7.38-$ $7.43(\mathrm{~m}, 2 \mathrm{H}) ;{ }^{13} \mathrm{C} \mathrm{NMR}\left(75 \mathrm{MHz}, \mathrm{CDCl}_{3}\right) \delta 14.2,22.7,26.4,27.9,29.3,31.5,50.3,54.1,70.6,83.0,123.4$, 127.6, 131.3, 155.9; IR (thin film) $\mathrm{cm}^{-1} 2928$ (m), 2240 (w), 1729 (s), 1441 (m), 1292 (m); mass spectrum (APCI): $\mathrm{m} / \mathrm{e}\left(\%\right.$ relative intensity) $260(100)(\mathrm{M}+\mathrm{H})^{+}$.

Ynamide 21 (56.0 mg, 58\% yield).

$\mathrm{R}_{\mathrm{f}}=0.44$ (20\% EtOAc in Hexanes); clear oil; ${ }^{1} \mathrm{H}$ NMR (300 MHz, $\left.\mathrm{CDCl}_{3}\right) \delta 1.23$ (s, $\left.6 \mathrm{H}\right), 2.00(\mathrm{t}, 2 \mathrm{H}, J=7.0$ $\mathrm{Hz}), 3.70$ (t, $2 \mathrm{H}, J=7.0 \mathrm{~Hz}), 7.23-7.34(\mathrm{~m}, 3 \mathrm{H}), 7.41-7.48(\mathrm{~m}, 2 \mathrm{H}) ;{ }^{13} \mathrm{C} \mathrm{NMR}\left(75 \mathrm{MHz}, \mathrm{CDCl}_{3}\right) \delta 24.5,34.6$, 39.8, 46.9, 72.2, 81.1, 122.8, 127.9, 128.3, 131.5, 180.7; IR (thin film) $\mathrm{cm}^{-1} 2965$ (w), 2241 (m), 1722 (s), 1398 (s); mass spectrum (APCI): m/e (\% relative intensity) $214(100)(\mathrm{M}+\mathrm{H})^{+}$.

Ynamide 22 (70.0 $\mathrm{mg}, 38 \%$ yield).

$\mathrm{R}_{\mathrm{f}}=0.38$ (20\% EtOAc in Hexanes); clear oil; ${ }^{1} \mathrm{H}$ NMR $\left(300 \mathrm{MHz}, \mathrm{CDCl}_{3}\right) \delta 2.15$ (quint, $2 \mathrm{H}, J=7.2 \mathrm{~Hz}$ ), 2.46 $(\mathrm{t}, 2 \mathrm{H}, J=7.2 \mathrm{~Hz}), 3.76(\mathrm{t}, 2 \mathrm{H}, J=7.2 \mathrm{~Hz}), 7.24-7.35(\mathrm{~m}, 3 \mathrm{H}), 7.39-7.49(\mathrm{~m}, 2 \mathrm{H}) ;{ }^{13} \mathrm{C} \mathrm{NMR}(75 \mathrm{MHz}$, $\mathrm{CDCl}_{3}$ ) $\delta 18.9,29.8,50.2,72.6,80.5,122.6,128.0,128.3,131.6,176.0$; IR (thin film) $\mathrm{cm}^{-1} 9279(\mathrm{w}), 2247(\mathrm{~m})$, $1721(\mathrm{~s}), 1397(\mathrm{~s})$; mass spectrum $(\mathrm{APCI}): \mathrm{m} / \mathrm{e}\left(\%\right.$ relative intensity) $186(100)(\mathrm{M}+\mathrm{H})^{+}$.

Ynamide 24 (109.0 mg, 97\% yield).

$\mathrm{R}_{\mathrm{f}}=0.46$ (14\% EtOAc in Hexanes); clear oil; ${ }^{1} \mathrm{H}$ NMR (300 MHz, $\left.\mathrm{CDCl}_{3}\right) \delta 2.49(\mathrm{~s}, 3 \mathrm{H}), 3.08(\mathrm{t}, 2 \mathrm{H}, J=7.8$ $\mathrm{Hz}), 3.74(\mathrm{t}, 2 \mathrm{H}, J=7.8 \mathrm{~Hz}), 7.23-7.50(\mathrm{~m}, 12 \mathrm{H}), 7.86(\mathrm{~d}, 2 \mathrm{H}, J=8.4 \mathrm{~Hz}) ;{ }^{13} \mathrm{C}$ NMR $\left(75 \mathrm{MHz}, \mathrm{CDCl}_{3}\right) \delta$; IR 
(thin film) $\mathrm{cm}^{-1} 3029$ (w), 2234 (m), 1598 (w), 1494 (w), 1365 (s), 1169 (s); mass spectrum (APCI): m/e (\% relative intensity) $376(100)(\mathrm{M}+\mathrm{H})^{+}$.

Ynamide 25 (287.0 mg, 93\% yield).

$\mathrm{R}_{\mathrm{f}}=0.41$ (50\% EtOAc in hexane); oil; ${ }^{1} \mathrm{H} \mathrm{NMR}\left(300 \mathrm{MHz}, \mathrm{CDCl}_{3}\right) \delta 2.38(\mathrm{~s}, 3 \mathrm{H}), 3.76(\mathrm{~s}, 3 \mathrm{H}), 4.57(\mathrm{~s}, 2 \mathrm{H})$, $6.76-6.83(\mathrm{~m}, 2 \mathrm{H}), 7.15-7.20(\mathrm{~m}, 2 \mathrm{H}), 7.24-7.29(\mathrm{~m}, 5 \mathrm{H}), 7.34-7.38(\mathrm{~m}, 2 \mathrm{H}), 7.83(\mathrm{~d}, 2 \mathrm{H}, J=8.4$ $\mathrm{Hz}) ;{ }^{13} \mathrm{C}$ NMR $\left(125 \mathrm{MHz}, \mathrm{CDCl}_{3}\right) \delta 21.5,55.5,55.7,67.7,86.5,110.6,111.9,120.2,127.7,128.1,128.3$, 128.8, 129.0, 129.5, 132.5, 134.5, 134.6, 144.5, 159.6; IR (thin film) cm $\mathrm{cm}^{-1} 3066$ (w), 2935 (w), 2237 (m), 1596 $(\mathrm{m}), 1497(\mathrm{~m}), 1364(\mathrm{~s}), 1170(\mathrm{~s})$; mass spectrum $(\mathrm{CI}): \mathrm{m} / \mathrm{e}\left(\%\right.$ relative intensity) $424(100)(\mathrm{M}+\mathrm{MeOH}+\mathrm{H})^{+}$, 392 (5), 378 (15), 269 (10), 236 (10).

Ynamide 26 (154.0 mg, 88\% yield).

$\mathrm{R}_{\mathrm{f}}=0.37$ (50\% EtOAc in hexane); oil; ${ }^{1} \mathrm{H} \mathrm{NMR}\left(300 \mathrm{MHz}, \mathrm{CDCl}_{3}\right) \delta 2.30(\mathrm{~s}, 3 \mathrm{H}), 3.83(\mathrm{~s}, 3 \mathrm{H}), 4.59(\mathrm{~s}, 2 \mathrm{H})$, $7.06(\mathrm{~d}, 2 \mathrm{H}, J=9.5 \mathrm{~Hz}), 7.20(\mathrm{~d}, 2 \mathrm{H}, J=8.0 \mathrm{~Hz}), 7.21(\mathrm{~m}, 1 \mathrm{H}), 7.25-7.28(\mathrm{~m}, 3 \mathrm{H}), 7.37-7.38(\mathrm{~m}, 2 \mathrm{H})$, $7.59-7.62(\mathrm{~m}, 3 \mathrm{H}), 7.85(\mathrm{~d}, 2 \mathrm{H}, J=8.5 \mathrm{~Hz}) ;{ }^{13} \mathrm{C} \mathrm{NMR}\left(125 \mathrm{MHz}, \mathrm{CDCl}_{3}\right) \delta$ 21.7, 55.9, 56.7, 66.5, 91.5, 104.8, 106.2, 113.0, 124.1, 125.3, 127.1, 127.2, 128.0, 128.4, 128.6, 128.7, 129.3, 129.8, 134.3, 134.7, 134.8, 144.7, 158.3; IR (thin film) cm $\mathrm{cm}^{-1} 3061$ (w), 2937 (w), 2235 (s), 1620 (m), 1591 (s), 1508 (s), 1364 (s), 1270 (s), 1170 (s); mass spectrum (CI): m/e (\% relative intensity) $442(50)(\mathrm{M}+\mathrm{H})^{+}, 391(25), 286(25), 186(100)$.

Ynamide 28 (106.0 $\mathrm{mg}, 75 \%$ yield).

$\mathrm{R}_{\mathrm{f}}=0.34$ (10\% EtOAc in Hexanes); clear oil; ${ }^{1} \mathrm{H}$ NMR $\left(300 \mathrm{MHz}, \mathrm{CDCl}_{3}\right) \delta 0.06(\mathrm{~s}, 6 \mathrm{H}), 0.92(\mathrm{~s}, 9 \mathrm{H}), 1.40-$ $1.55(\mathrm{~m}, 4 \mathrm{H}), 2.22(\mathrm{t}, 2 \mathrm{H}, J=6.6 \mathrm{~Hz}), 2.47(\mathrm{~s}, 3 \mathrm{H}), 3.56(\mathrm{t}, 2 \mathrm{H}, J=6.0 \mathrm{~Hz}), 4.47(\mathrm{~s}, 2 \mathrm{H}), 7.33(\mathrm{~d}, 2 \mathrm{H}, J=$ $6.6 \mathrm{~Hz}), 7.78(\mathrm{~d}, 2 \mathrm{H}, J=6.6 \mathrm{~Hz}) ;{ }^{13} \mathrm{C} \mathrm{NMR}\left(75 \mathrm{MHz}, \mathrm{CDCl}_{3}\right) \delta-5.2,18.3,18.5,21.8,25.4,26.1,31.8,55.7$, $62.7,70.8,73.6,127.8,128.2,128.5,128.9,129.7,134.8,134.9,144.4$; IR (thin film) $\mathrm{cm}^{-1} 2930(\mathrm{~m}), 2857(\mathrm{~m})$, $2246(\mathrm{w}), 1365(\mathrm{~m}), 1170(\mathrm{~m}), 1092(\mathrm{~m})$; mass spectrum (APCI): m/e (\% relative intensity) $472(100)(\mathrm{M}+\mathrm{H})^{+}$.

Ynamide 29 (154.0 $\mathrm{mg}, 74 \%$ yield).

$\mathrm{R}_{\mathrm{f}}=0.48$ (25\% EtOAc in hexanes); yellow solid; ${ }^{1} \mathrm{H}$ NMR $\left(300 \mathrm{MHz}, \mathrm{CDCl}_{3}\right): \delta 1.01(\mathrm{~s}, 9 \mathrm{H}), 2.42(\mathrm{~s}, 3 \mathrm{H})$, 4.36 (s, $2 \mathrm{H}), 4.40$ (s, $2 \mathrm{H}), 7.18-7.28$ (m, $7 \mathrm{H}), 7.28-7.36$ (ddt, $4 \mathrm{H}, J=1.4,7.2,8.4 \mathrm{~Hz}$ ), 7.36-7.44 (ddt, $2 \mathrm{H}, J$ $=1.5,7.4,7.2), 7.61-7.64(\mathrm{~m}, 4 \mathrm{H}), 7.72-7.74(\mathrm{~d}, 2 \mathrm{H}, J=8.4 \mathrm{~Hz}) ;{ }^{13} \mathrm{C} \mathrm{NMR}\left(75 \mathrm{MHz}, \mathrm{CDCl}_{3}\right) \delta 19.1,21.5$, 26.6 (3 C), 52.7, 55.3, 70.1, 78.6, 127.5 (6 C), 128.0, 128.3 (2 C), 128.4 (2 C), 129.5 (2, C), 129.6 (2 C), 133.1 (2 C), 134.4, 134.7, 135.5 (4 C), 144.3. IR (thin film) cm $\mathrm{cm}^{-1} 3069$ (m), 2246 (m), 1596 (w), 1495 (w), 1367 (s), 1111 (s), 1074 (s); mass spectrum (ESI): m/e (\% relative intensity) 576.1 (100) $\left(\mathrm{M}+\mathrm{Na}^{+}\right)$; m/e calcd for $\mathrm{C}_{33} \mathrm{H}_{35} \mathrm{NO}_{3} \mathrm{SSiNa}\left(\mathrm{M}+\mathrm{Na}^{+}\right): 576.1999$, found: $576.2003 ;(\alpha)_{\mathrm{D}}{ }^{23}=(-) 10.831\left(c=0.67, \mathrm{CH}_{2} \mathrm{Cl}_{2}\right)$.

Ynamide 30 ( $852.0 \mathrm{mg}, 97 \%$ yield).

$\mathrm{R}_{\mathrm{f}}=0.53$ (10\% EtOAc in Hexanes); clear oil; ${ }^{1} \mathrm{H}$ NMR $\left(300 \mathrm{MHz}, \mathrm{CDCl}_{3}\right) \delta 0.94(\mathrm{~s}, 21 \mathrm{H}), 2.40(\mathrm{~s}, 3 \mathrm{H}), 4.48$ $(\mathrm{s}, 2 \mathrm{H}), 7.20-7.30(\mathrm{~m}, 7 \mathrm{H}), 7.74(\mathrm{~d}, 2 \mathrm{H}, J=6.6 \mathrm{~Hz}) ;{ }^{13} \mathrm{C} \mathrm{NMR}\left(75 \mathrm{MHz}, \mathrm{CDCl}_{3}\right) \delta 11.3,18.5,21.6,55.5$, 
70.2, 96.5, 127.8, 128.3, 128.5, 129.0, 129.6, 134.4, 134.6, 144.6; IR (thin film) $\mathrm{cm}^{-1} 2942(\mathrm{~m}), 2864$ (m), 2164 (m), $1370(\mathrm{~m}), 1170(\mathrm{~s})$; mass spectrum (APCI): m/e (\% relative intensity) $442(100)(\mathrm{M}+\mathrm{H})^{+}$.

Ynamide 31 (103.0 mg, 92\% yield).

$\mathrm{R}_{\mathrm{f}}=0.49\left(14 \%\right.$ EtOAc in Hexanes); clear oil; $(\alpha)_{\mathrm{D}}{ }^{23}=51.528\left(c=2.29, \mathrm{CH}_{2} \mathrm{Cl}_{2}\right)$;

${ }^{1} \mathrm{H}$ NMR $\left(300 \mathrm{MHz}, \mathrm{CDCl}_{3}\right) \delta 1.56(\mathrm{~d}, 3 \mathrm{H} \mathrm{J}=7.2 \mathrm{~Hz}), 2.34(\mathrm{~s}, 3 \mathrm{H}), 5.23(\mathrm{q}, 1 \mathrm{H}, J=7.2 \mathrm{~Hz}), 7.15-7.38(\mathrm{~m}$, $12 \mathrm{H}), 7.66(\mathrm{~d}, 2 \mathrm{H}, J=8.4 \mathrm{~Hz}) ;{ }^{13} \mathrm{C} \mathrm{NMR}\left(75 \mathrm{MHz}, \mathrm{CDCl}_{3}\right) \delta$ 19.7, 21.6, 59.0, 73.4, 80.4, 123.1, 126.9, 127.6, 127.7, 128.0, 128.3, 128.4, 129.5, 131.1, 135.4, 139.8, 144.4; IR (thin film) cm $\mathrm{cm}^{-1} 2988(\mathrm{w}), 2233$ (m), 1364 (s), 1170 (s); mass spectrum (APCI): m/e (\% relative intensity) $376(100)(\mathrm{M}+\mathrm{H})^{+}, 221(22), 105(14)$.

Ynamide 32 (101.0 mg, 97\% yield).

$\mathrm{R}_{\mathrm{f}}=0.41$ (14\% EtOAc in Hexanes); mp 66-68 ${ }^{\circ} \mathrm{C} ;{ }^{1} \mathrm{H}$ NMR $\left(300 \mathrm{MHz}, \mathrm{CDCl}_{3}\right) \delta 4.59$ (s, $\left.3 \mathrm{H}\right), 7.20-7.38(\mathrm{~m}, 10$ $\mathrm{H}), 7.50(\mathrm{t}, 2 \mathrm{H}, J=7.8 \mathrm{~Hz}), 7.61(\mathrm{t}, 1 \mathrm{H} J=7.8 \mathrm{~Hz}), 7.90(\mathrm{~d}, 2 \mathrm{H}, J=7.8 \mathrm{~Hz}) ;{ }^{13} \mathrm{C} \mathrm{NMR}\left(75 \mathrm{MHz} \mathrm{CDCl}_{3}\right) \delta$ 55.8, 71.4, 82.6, 122.7, 127.7. 127.9, 128.3, 128.4, 128.6, 128.9, 129.2, 131.2, 133.7, 134.3, 137.6; IR (thin film) $\mathrm{cm}^{-1} 3064(\mathrm{w}), 2235(\mathrm{~m}), 1447(\mathrm{~m}), 1365(\mathrm{~s}), 1172(\mathrm{~s}), 1090(\mathrm{~m}), 752$ (s); mass spectrum (APCI): m/e (\% relative intensity) $380(100)(\mathrm{M}+\mathrm{MeOH}+\mathrm{H})^{+}, 348(14)(\mathrm{M}+\mathrm{H})^{+}, 239(12), 91$ (21).

Ynamide 33 (151.0 mg, 91\% yield).

$\mathrm{R}_{\mathrm{f}}=0.28$ (14\% EtOAc in Hexanes); mp 78-82 ${ }^{\circ} \mathrm{C} ;{ }^{1} \mathrm{H}$ NMR $\left(300 \mathrm{MHz}, \mathrm{CDCl}_{3}\right) \delta$ 7.24-7.41 (m, $\left.10 \mathrm{H}\right), 7.48(\mathrm{t}, 2$ $\mathrm{H}, J=7.5 \mathrm{~Hz}), 7.63(\mathrm{t}, 1 \mathrm{H}, J=7.5 \mathrm{~Hz}), 7.74(\mathrm{~d}, 2 \mathrm{H}, J=7.5 \mathrm{~Hz}) ;{ }^{13} \mathrm{C} \mathrm{NMR}\left(75 \mathrm{MHz}, \mathrm{CDCl}_{3}\right) \delta 70.6,82.9$, $122.5,126.3,128.2,128.3,128.4,128.5,129.0,129.3,131.6,134.1,135.8,138.9$; IR (thin film) $\mathrm{cm}^{-1} 3060(\mathrm{w})$, $2240(\mathrm{~m}), 1490(\mathrm{~m}), 1448(\mathrm{~m}), 1374(\mathrm{~s}), 1173(\mathrm{~s})$; mass spectrum (APCI): m/e (\% relative intensity) 366 (100) $(\mathrm{M}+\mathrm{MeOH}+\mathrm{H})^{+}, 334(53)(\mathrm{M}+\mathrm{H})^{+}, 193(87)$.

Ynamide 34 (128.0 mg, 94\% yield).

$\mathrm{R}_{\mathrm{f}}=0.35\left(14 \%\right.$ EtOAc in Hexanes); clear oil; $(\alpha)_{\mathrm{D}}{ }^{23}=86.806\left(c=1.91, \mathrm{CH}_{2} \mathrm{Cl}_{2}\right)$;

${ }^{1} \mathrm{H}$ NMR $\left(300 \mathrm{MHz}, \mathrm{CDCl}_{3}\right) \delta 1.59(\mathrm{~d}, 3 \mathrm{H}, J=7.2 \mathrm{~Hz}), 5.24(\mathrm{q}, 1 \mathrm{H}, J=7.2 \mathrm{~Hz}), 7.19-7.37(\mathrm{~m}, 10 \mathrm{H}), 7.39(\mathrm{t}$, $2 \mathrm{H}, J=7.5 \mathrm{~Hz}), 7.54(\mathrm{t}, 1 \mathrm{H}, J=7.5 \mathrm{~Hz}), 7.76(\mathrm{~d}, 2 \mathrm{H}, J=7.5 \mathrm{~Hz}) ;{ }^{13} \mathrm{C} \mathrm{NMR}\left(75 \mathrm{MHz}, \mathrm{CDCl}_{3}\right) \delta 19.8,59.2$, 73.4, 80.3, 123.0, 126.9, 127.6, 127.8, 128.2, 128.4, 128.5, 128.9, 131.2, 133.4, 138.2, 139.7; IR (thin film) $\mathrm{cm}^{-1}$ $2233(\mathrm{~m}), 1447(\mathrm{~m}), 1364$ (s), 1172 (s); mass spectrum (APCI): m/e (\% relative intensity) $362(100)(\mathrm{M}+\mathrm{H})^{+}$, $221(45)$;.

Ynamide 35 (111.0 mg, 98\% yield).

$\mathrm{R}_{\mathrm{f}}=0.33$ (20\% EtOAc in Hexanes); clear oil; ${ }^{1} \mathrm{H} \mathrm{NMR}\left(300 \mathrm{MHz}, \mathrm{CDCl}_{3}\right) \delta 3.86(\mathrm{~s}, 3 \mathrm{H}), 4.63(\mathrm{~s}, 3 \mathrm{H}), 7.01$ $(\mathrm{d}, 2 \mathrm{H}, J=9.0 \mathrm{~Hz}), 7.23-7.42(\mathrm{~m}, 10 \mathrm{H}), 7.90(\mathrm{~d}, 2 \mathrm{H}, J=9.0 \mathrm{~Hz}) ;{ }^{13} \mathrm{C} \mathrm{NMR}\left(75 \mathrm{MHz}, \mathrm{CDCl}_{3}\right) \delta 55.6,55.7$, $71.3,82.9,114.3,122.8,127.7,128.2,128.3,128.5,128.8,129.0,129.9,131.1,134.5,163.7$; IR (thin film) $\mathrm{cm}^{-1}$ 3060 (w), 2232 (m), 1595 (s), 1497 (s), 1363 (s), 1263 (s), 1164 (s), 1092 (m), 1023 (m); mass spectrum (APCI): m/e (\% relative intensity) $410(100)(\mathrm{M}+\mathrm{MeOH}+\mathrm{H})^{+}, 378(5)(\mathrm{M}+\mathrm{H})^{+}, 171(12), 91(22)$. 
Ynamide 36 (114.0 mg, 97\% yield).

$\mathrm{R}_{\mathrm{f}}=0.35$ (33\% EtOAc in Hexanes); mp 105-108 ${ }^{\circ} \mathrm{C} ;{ }^{1} \mathrm{H}$ NMR (300 MHz, $\left.\mathrm{CDCl}_{3}\right) \delta 4.71$ (s, $\left.3 \mathrm{H}\right), 7.26-7.42(\mathrm{~m}$, $10 \mathrm{H}), 8.01(\mathrm{~d}, 2 \mathrm{H}, J=8.7 \mathrm{~Hz}), 8.30(\mathrm{~d}, 2 \mathrm{H}, J=8.7 \mathrm{~Hz}) ;{ }^{13} \mathrm{C} \mathrm{NMR}\left(75 \mathrm{MHz}, \mathrm{CDCl}_{3}\right) \delta 56.4,71.8,81.7,122.0$, $124.2,128.3,128.4,128.7,128.8,128.9,129.0,131.4,133.8,142.9,150.4$; IR (thin film) $\mathrm{cm}^{-1} 2238$ (m), 1531 (s), $1373(\mathrm{~s}), 1349$ (s), $1174(\mathrm{~s})$; mass spectrum (APCI): m/e (\% relative intensity) $425(98)(\mathrm{M}+\mathrm{MeOH}+\mathrm{H})^{+}$, $393(11)(\mathrm{M}+\mathrm{H})^{+}, 239(38), 91(100)$.

Ynamide 37 ( $83.0 \mathrm{mg}, 97 \%$ yield).

$\mathrm{R}_{\mathrm{f}}=0.32$ (20\% EtOAc in Hexanes); mp 55-56 ${ }^{\circ} \mathrm{C} ;{ }^{1} \mathrm{H}$ NMR (300 MHz, $\left.\mathrm{CDCl}_{3}\right) \delta 3.93(\mathrm{~s}, 3 \mathrm{H}), 4.70(\mathrm{~s}, 3 \mathrm{H})$, 7.23-7.54 (m, $10 \mathrm{H}) ;{ }^{13} \mathrm{C} \mathrm{NMR}\left(75 \mathrm{MHz}, \mathrm{CDCl}_{3}\right) \delta 38.9,55.9,71.6,82.1,122.5,128.1,128.4,128.8,128.9$, 129.0, 131.4, 134.6; IR (thin film) $\mathrm{cm}^{-1} 3032$ (w), 2235 (m), 1358 (s), 1164 (s); mass spectrum (APCI): m/e (\% relative intensity) $318(100),(\mathrm{M}+\mathrm{MeOH}+\mathrm{H})^{+}, 286(43)(\mathrm{M}+\mathrm{H})^{+}, 239$ (27), 91 (66).

Ynamide 38 (64.0 $\mathrm{mg}, 69 \%$ yield).

$\mathrm{R}_{\mathrm{f}}=0.47$ (14\% EtOAc in Hexanes); clear oil; ${ }^{1} \mathrm{H}$ NMR $\left(300 \mathrm{MHz}, \mathrm{CDCl}_{3}\right) \delta 2.44(\mathrm{~s}, 3 \mathrm{H}), 4.07(\mathrm{~d}, 2 \mathrm{H}, J=6.6$ $\mathrm{Hz}), 5.27(\mathrm{~d}, 1 \mathrm{H}, J=10.4 \mathrm{~Hz}$ ), $5.34(\mathrm{~d}, 1 \mathrm{H}, J=16.6 \mathrm{~Hz}$ ), 5.82 (ddt, $1 \mathrm{H}, J=6.6,10.4,16.6 \mathrm{~Hz}), 7.28-7.43(\mathrm{~m}$, $7 \mathrm{H}), 7.85(\mathrm{~d}, 2 \mathrm{H}, J=8.4 \mathrm{~Hz}) ;{ }^{13} \mathrm{C} \mathrm{NMR}\left(75 \mathrm{MHz}, \mathrm{CDCl}_{3}\right) \delta 21.6,54.4,70.9,82.3,120.1,122.8,127.7,128.2$, 128.7, 129.8, 130.9, 131.3, 134.6, 144.8; IR (thin film) cm $\mathrm{cm}^{-1} 3062(\mathrm{w}), 2923(\mathrm{w}), 2235$ (m), 1597 (m), $1364(\mathrm{~s})$, 1170 (s); mass spectrum (APCI): m/e (\% relative intensity) $344(100)(\mathrm{M}+\mathrm{MeOH}+\mathrm{H})^{+}, 312(17)(\mathrm{M}+\mathrm{H})^{+}$, $156(21)$.

Ynamide 39 (197.0 mg, 92\% yield).

$\mathrm{R}_{\mathrm{f}}=0.48\left(10 \%\right.$ EtOAc in Hexanes); clear oil; ${ }^{1} \mathrm{H} \mathrm{NMR}\left(300 \mathrm{MHz}, \mathrm{CDCl}_{3}\right) \delta 0.07(\mathrm{~s}, 6 \mathrm{H}), 0.89(\mathrm{~s}, 9 \mathrm{H}), 2.44$ $(\mathrm{s}, 3 \mathrm{H}), 3.56$ (t, $2 \mathrm{H}, J=6.0 \mathrm{~Hz}), 3.89(\mathrm{t}, 2 \mathrm{H}, J=6.0 \mathrm{~Hz}), 7.23-7.40(\mathrm{~m}, 7 \mathrm{H}), 7,87(\mathrm{~d}, 2 \mathrm{H}, J=8.1 \mathrm{~Hz}) ;{ }^{13} \mathrm{C}$ $\operatorname{NMR}\left(75 \mathrm{MHz}, \mathrm{CDCl}_{3}\right) \delta-5.3,18.4,21.7,25.9,53.7,60.6,70.3,82.7,123.0,127.8,127.9,128.3,129.8,131.4$, 134.9, 144.7; IR (thin film) $\mathrm{cm}^{-1} 2930(\mathrm{~m}), 2235$ (m), 1367 (s), 1171 (s), 1104 (s); mass spectrum (APCI): m/e (\% relative intensity) $462(100)(\mathrm{M}+\mathrm{MeOH}+\mathrm{H})^{+}, 430(5)(\mathrm{M}+\mathrm{H})^{+}$.

Ynamide 40 (76.0 $\mathrm{mg}, 92 \%$ yield).

$\mathrm{R}_{\mathrm{f}}=0.43$ (10\% EtOAc in Hexanes); mp 95-97 ${ }^{\circ} \mathrm{C} ;{ }^{1} \mathrm{H} \mathrm{NMR}\left(300 \mathrm{MHz}, \mathrm{CD}_{2} \mathrm{Cl}_{2}\right) \delta 3.93(\mathrm{~s}, 3 \mathrm{H}), 7.37-7.46(\mathrm{~m}$, $5 \mathrm{H}), 7.58-7.64(\mathrm{~m}, 2 \mathrm{H}), 7.65(\mathrm{~d}, 1 \mathrm{H}, J=8.1 \mathrm{~Hz}), 7.94(\mathrm{~s}, 1 \mathrm{H}), 8.21(\mathrm{~d}, 1 \mathrm{H}, J=8.1 \mathrm{~Hz}) ;{ }^{13} \mathrm{C} \mathrm{NMR}(75 \mathrm{MHz}$, $\left.\mathrm{CD}_{2} \mathrm{Cl}_{2}\right) \delta 51.8,72.3,79.6,111.5,112.0,122.2,122.3,124.2,125.0,129.1,129.2,132.0,135.2,138.9,164.5 ;$ IR (thin film) $\mathrm{cm}^{-1} 2256(\mathrm{~m}), 1711(\mathrm{~s}), 1543(\mathrm{~s}), 1458$ (s), 1195 (s); mass spectrum (APCI): m/e (\% relative intensity) $276(100)(\mathrm{M}+\mathrm{H})^{+}$.

Ynamide 41 (100.0 mg, 93\% yield).

$\mathrm{R}_{\mathrm{f}}=0.58$ (10\% EtOAc in Hexanes); mp 54-56 ${ }^{\circ} \mathrm{C} ;{ }^{1} \mathrm{H}$ NMR (300 MHz, $\left.\mathrm{CD}_{2} \mathrm{Cl}_{2}\right) \delta 1.18-1.27(\mathrm{~s}, 21 \mathrm{H}), 3.92(\mathrm{~s}, 3$ $\mathrm{H}), 7.38(\mathrm{t}, 1 \mathrm{H}, J=7.8 \mathrm{~Hz}), 7.41(\mathrm{t}, 1 \mathrm{H}, J=7.8 \mathrm{~Hz}), 7.61(\mathrm{~d}, 1 \mathrm{H}, J=7.8 \mathrm{~Hz}), 7.96(\mathrm{~s}, 1 \mathrm{H}), 8.18(\mathrm{~d}, 1 \mathrm{H}, J=$ $7.8 \mathrm{~Hz}) ;{ }^{13} \mathrm{C} \mathrm{NMR}\left(75 \mathrm{MHz}, \mathrm{CD}_{2} \mathrm{Cl}_{2}\right) \delta 11.9,19.1,51.8,71.4,93.4,111.3,112.1,122.4,124.2,125.2,125.9$, 
135.5, 139.1, 164.6; IR (thin film) $\mathrm{cm}^{-1} 2944$ (w), 2865 (s), 2183 (s), 1718 (s), 1543 (s), 1458 (s), 1206 (s); mass spectrum (APCI): m/e (\% relative intensity) $388(100)(\mathrm{M}+\mathrm{MeOH}+\mathrm{H})^{+}, 356(51)(\mathrm{M}+\mathrm{H})^{+}$.

Ynamide 42 (72.0 mg, 85\% yield).

$\mathrm{R}_{\mathrm{f}}=0.54$ (10\% EtOAc in Hexanes); clear oil;

${ }^{1} \mathrm{H}$ NMR $\left(300 \mathrm{MHz}, \mathrm{CD}_{2} \mathrm{Cl}_{2}\right) \delta 0.95(\mathrm{t}, 3 \mathrm{H}, J=6.9 \mathrm{~Hz}), 1.30-1.42(\mathrm{~m}, 4 \mathrm{H}), 1.42-1.60(\mathrm{~m}, 2 \mathrm{H}), 1.60-1.74$ (quintet, $2 \mathrm{H}, J=6.9 \mathrm{~Hz}), 2.49(\mathrm{t}, 2 \mathrm{H}, J=6.9 \mathrm{~Hz}$ ), $3.90(\mathrm{~s}, 3 \mathrm{H}), 7.36$ (quintet, $2 \mathrm{H}, J=7.5 \mathrm{~Hz}), 7.56(\mathrm{~d}, 1 \mathrm{H}, J$ $=7.5 \mathrm{~Hz}), 7.88(\mathrm{~s}, 1 \mathrm{H}), 8.16(\mathrm{~d}, 1 \mathrm{H}, J=7.5 \mathrm{~Hz}) ;{ }^{13} \mathrm{C} \mathrm{NMR}\left(75 \mathrm{MHz}, \mathrm{CD}_{2} \mathrm{Cl}_{2}\right) \delta 14.4,18.8,23.2,29.2,29.3$, $32.0,51.7,70.9,72.5,110.4,111.9,122.2,123.8,124.7,125.8,135.6,139.1,164.7$; IR (thin film) $\mathrm{cm}^{-1} 2931$ (m), $2270(\mathrm{w}), 1714(\mathrm{~s}), 1542(\mathrm{~m}), 1462(\mathrm{~m}), 1210(\mathrm{~s})$; mass spectrum (APCI): m/e (\% relative intensity) 316 (100) $(\mathrm{M}+\mathrm{MeOH}+\mathrm{H})^{+}, 284(73)(\mathrm{M}+\mathrm{H})^{+}$.

Ynamide 43 (62.0 mg, 80\% yield).

$\mathrm{R}_{\mathrm{f}}=0.30$ (14\% EtOAc in Hexanes); $\mathrm{mp} 95-97^{\circ} \mathrm{C}$;

${ }^{1} \mathrm{H}$ NMR $\left(300 \mathrm{MHz}, \mathrm{CD}_{2} \mathrm{Cl}_{2}\right) \delta 2.53(\mathrm{~s}, 3 \mathrm{H}), 7.38-7.52(\mathrm{~m}, 5 \mathrm{H}), 7.57-7.73(\mathrm{~m}, 3 \mathrm{H}), 7.91(\mathrm{~s}, 1 \mathrm{H}), 8.37(\mathrm{~d}, 1 \mathrm{H}$, $J=7.5 \mathrm{~Hz}) ;{ }^{13} \mathrm{C}$ NMR $\left(75 \mathrm{MHz}, \mathrm{CD}_{2} \mathrm{Cl}_{2}\right) \delta 28.0,72.3,79.6,111.8,120.2,122.1,123.2,124.6,125.4,125.6$, 129.1, 129.2, 132.0, 135.9, 139.0, 193.2; IR (thin film) $\mathrm{cm}^{-1} 3055$ (w), 2255 (m), 1661 (s), 1534 (s), 1457 (s), 1190 (s); mass spectrum (APCI): m/e (\% relative intensity) $260(100)(\mathrm{M}+\mathrm{H})^{+}$.

Ynamide 44 (46.0 $\mathrm{mg}, 72 \%$ yield).

$\mathrm{R}_{\mathrm{f}}=0.45$ (14\% EtOAc in Hexanes); mp $127-129^{\circ} \mathrm{C}$;

${ }^{1} \mathrm{H}$ NMR $\left(300 \mathrm{MHz}, \mathrm{CD}_{2} \mathrm{Cl}_{2}\right) \delta$ 7.40-7.47 (m, $\left.3 \mathrm{H}\right), 7.48(\mathrm{t}, 1 \mathrm{H}, J=1.8 \mathrm{~Hz}), 7.50-7.67(\mathrm{~m}, 6 \mathrm{H}), 7.75(\mathrm{~d}, 1 \mathrm{H}, J$ $=8.4 \mathrm{~Hz}), 7.82(\mathrm{~s}, 1 \mathrm{H}), 7.90(\mathrm{~d}, 2 \mathrm{H}, J=8.4 \mathrm{~Hz}), 8.43(\mathrm{~d}, 1 \mathrm{H}, J=8.4 \mathrm{~Hz}) ;{ }^{13} \mathrm{C} \mathrm{NMR}\left(75 \mathrm{MHz} \mathrm{CD}_{2} \mathrm{Cl}_{2}\right) \delta$ 72.4, 79.6, 111.8, 118.9, 122.0, 123.3, 124.7, 125.7, 126.6, 129.0, 129.1, 129.3, 132.0, 132.3, 137.7, 139.1, 140.4, 190.7; IR (thin film) $\mathrm{cm}^{-1} 3054$ (w), 2254 (m), 1634 (s), 1528 (s), 1457 (s), 1207 (s); mass spectrum (APCI): $\mathrm{m} / \mathrm{e}\left(\%\right.$ relative intensity) $322(100)(\mathrm{M}+\mathrm{H})^{+}$.

Ynamide 45 (70.0 $\mathrm{mg}, 81 \%$ yield).

$\mathrm{R}_{\mathrm{f}}=0.57$ (10\% EtOAc in Hexanes); mp 52-53 ${ }^{\circ} \mathrm{C}$;

${ }^{1} \mathrm{H}$ NMR (300 MHz, $\left.\mathrm{CD}_{2} \mathrm{Cl}_{2}\right) \delta 1.27(\mathrm{t}, 3 \mathrm{H}, J=7.2 \mathrm{~Hz}), 4.27(\mathrm{q}, 2 \mathrm{H}, J=7.2 \mathrm{~Hz}), 7.14(\mathrm{t}, 1 \mathrm{H}, J=7.5 \mathrm{~Hz})$, 7.20-7.30 (m, $4 \mathrm{H}), 7.33(\mathrm{t}, 1 \mathrm{H}, J=7.8 \mathrm{~Hz}), 7.45-7.52(\mathrm{~m}, 2 \mathrm{H}), 7.56(\mathrm{t}, 2 \mathrm{H}, J=7.8 \mathrm{~Hz}) ;{ }^{13} \mathrm{C} \mathrm{NMR}(75 \mathrm{MHz}$, $\left.\mathrm{CD}_{2} \mathrm{Cl}_{2}\right) \delta 14.7,61.7,73.6,80.1,112.6,113.4,123.2,123.3,123.7,126.7,127.2,128.6,129.0,130.0,131.8$, 141.0, 160.6; IR (thin film) $\mathrm{cm}^{-1} 2983(\mathrm{w}), 2256(\mathrm{~m}), 1720(\mathrm{~s}), 1451(\mathrm{~m}), 1271(\mathrm{~m}), 1196$ (s); mass spectrum (APCI): $\mathrm{m} / \mathrm{e}$ (\% relative intensity) $290(100)(\mathrm{M}+\mathrm{H})^{+}, 262(22)$.

Ynamide 46 (35.0 mg, 50\% yield).

$\mathrm{R}_{\mathrm{f}}=0.43\left(14 \%\right.$ EtOAc in Hexanes); mp 71-73 ${ }^{\circ} \mathrm{C} ;{ }^{1} \mathrm{H}$ NMR $\left(300 \mathrm{MHz}, \mathrm{CD}_{2} \mathrm{Cl}_{2}\right) \delta 2.24(\mathrm{~s}, 3 \mathrm{H}), 2.42(\mathrm{~s}, 3 \mathrm{H})$, $2.62(\mathrm{~s}, 3 \mathrm{H}), 6.64(\mathrm{~d}, 1 \mathrm{H}, J=1.2 \mathrm{~Hz}), 7.30-7.45(\mathrm{~m}, 3 \mathrm{H}), 7.46-7.60(\mathrm{~m}, 2 \mathrm{H}) ;{ }^{13} \mathrm{C} \mathrm{NMR}\left(75 \mathrm{MHz}, \mathrm{CD}_{2} \mathrm{Cl}_{2}\right) \delta$ 13.6, 31.5, 72.1, 80.5, 121.7, 121.8, 122.4, 123.0, 129.0, 129.1, 131.8, 139.6, 195.1; IR (thin film) $\mathrm{cm}^{-1} 2938$ 
(w), $2247(\mathrm{~m}), 1656(\mathrm{~s}), 1514(\mathrm{~m}), 1418(\mathrm{~s}) ;$ mass spectrum (APCI): m/e (\% relative intensity) $238(100)(\mathrm{M}+$ $\mathrm{H})^{+}$.

Ynamide 47 (58.0 $\mathrm{mg}, 71 \%$ yield).

$\mathrm{R}_{\mathrm{f}}=0.43$ (33\% EtOAc in Hexanes); clear oil; ${ }^{1} \mathrm{H}$ NMR $\left(300 \mathrm{MHz}, \mathrm{CD}_{2} \mathrm{Cl}_{2}\right) \delta 6.79$ (dd, $\left.1 \mathrm{H}, J=1.5,3.0 \mathrm{~Hz}\right)$, $7.04(\mathrm{dd}, 1 \mathrm{H}, J=2.1,3.0 \mathrm{~Hz}), 7.35-7.43(\mathrm{~m}, 3 \mathrm{H}), 7.47-7.64(\mathrm{~m}, 6 \mathrm{H}), 7.83-7.90(\mathrm{~m}, 2 \mathrm{H}) ;{ }^{13} \mathrm{C} \mathrm{NMR}(75 \mathrm{MHz}$, $\left.\mathrm{CD}_{2} \mathrm{Cl}_{2}\right) \delta 68.4,82.0,112.2,121.8,126.3,126.6,128.9,129.1,129.3,129.4,131.4,132.0,132.4,139.8,189.8 ;$ IR (thin film) $\mathrm{cm}^{-1} 3131$ (w), 3059 (w), 2260 (m), 1641 (s), 1485 (s), 1285(s), 1122 (m); ); mass spectrum (APCI): $\mathrm{m} / \mathrm{e}\left(\%\right.$ relative intensity) $272(100)(\mathrm{M}+\mathrm{H})^{+}$.

Ynamide 48 (54.0 $\mathrm{mg}, 72 \%$ yield).

$\mathrm{R}_{\mathrm{f}}=0.31$ (20\% EtOAc in Hexanes); mp 102-104 ${ }^{\circ} \mathrm{C} ;{ }^{1} \mathrm{H} \mathrm{NMR}\left(300 \mathrm{MHz}, \mathrm{CDCl}_{3}\right.$ ) $\delta 2.18$ (quintet, $2 \mathrm{H}, J=6.3$ $\mathrm{Hz}), 2.49$ (t, $2 \mathrm{H}, J=6.3 \mathrm{~Hz}), 2.91(\mathrm{t}, 2 \mathrm{H}, J=6.3 \mathrm{~Hz}), 6.58(\mathrm{~d}, 1 \mathrm{H}, J=3.3 \mathrm{~Hz}), 6.84(\mathrm{~d}, 1 \mathrm{H}, J=3.3 \mathrm{~Hz}), 7.32-$ $7.41(\mathrm{~m}, 3 \mathrm{H}), 7.43-7.55(\mathrm{~m}, 2 \mathrm{H}) ;{ }^{13} \mathrm{C} \mathrm{NMR}\left(75 \mathrm{MHz}, \mathrm{CDCl}_{3}\right) \delta$ 22.0, 23.3, 37.7, 70.8, 79.5, 107.2, 121.4, 121.5, 124.9, 128.6, 128.9, 131.7, 147.3, 193.9; IR (thin film) $\mathrm{cm}^{-1} 3115$ (w), 2948 (w), 2259 (m), 1668 (s), 1459 (s); mass spectrum (APCI): m/e (\% relative intensity) $236(100)(\mathrm{M}+\mathrm{H})^{+}$.

Ynamide 49 (50.0 $\mathrm{mg}, 67 \%$ yield).

$\mathrm{R}_{\mathrm{f}}=0.43\left(33 \%\right.$ EtOAc in Hexanes); clear oil; ${ }^{1} \mathrm{H} \mathrm{NMR}\left(300 \mathrm{MHz}, \mathrm{CDCl}_{3}\right) \delta 3.45(\mathrm{~s}, 3 \mathrm{H}), 6.78-7.23(\mathrm{~m}, 1 \mathrm{H})$, 7.17-7.25 (m, $2 \mathrm{H})$, 7.26-7.32 (m, $1 \mathrm{H}), 7.34-7.43(\mathrm{~m}, 3 \mathrm{H}), 7.56-7.64(\mathrm{~m}, 2 \mathrm{H}) ;{ }^{13} \mathrm{C} \mathrm{NMR}\left(75 \mathrm{MHz}, \mathrm{CDCl}_{3}\right) \delta$ 27.7, 75.3, 75.5, 108.1, 109.9, 122.1, 122.3, 123.6, 128.2, 128.4, 128.6, 129.8, 131.9, 153.2; IR (thin film) $\mathrm{cm}^{-1}$ 3061 (w), 2252 (m), 1738 (s), 1492 (m), 1431 (m), 1413 (m); mass spectrum (APCI): m/e (\% relative intensity) $249(100)(\mathrm{M}+\mathrm{H})^{+}$.

Ynamide 50 (61.0 $\mathrm{mg}, 62 \%$ yield).

$\mathrm{R}_{\mathrm{f}}=0.51$ (20\% EtOAc in Hexanes); mp 77-78 ${ }^{\circ}$; ${ }^{1} \mathrm{H}$ NMR $\left(300 \mathrm{MHz}, \mathrm{CDCl}_{3}\right) \delta 1.16(\mathrm{~s}, 21 \mathrm{H}), 3.40(\mathrm{~s}, 3 \mathrm{H})$, 6.92-7.00 (m, $1 \mathrm{H})$, 7.15-7.25 (m, $3 \mathrm{H}) ;{ }^{13} \mathrm{C} \mathrm{NMR}\left(75 \mathrm{MHz}, \mathrm{CDCl}_{3}\right.$ ) $\delta$ 11.4, 18.8, 27.6, 74.5, 89.2, 108.0, 110.0, 122.3, 123.6, 128.3, 129.7, 153.4; IR (thin film) $\mathrm{cm}^{-1} 2941$ (s), 2864 (s), 2184 (s), 1742 (s), 1398 (s), 731 (s); mass spectrum (APCI): m/e (\% relative intensity) $361(100)(\mathrm{M}+\mathrm{MeOH}+\mathrm{H})^{+}, 329(11)(\mathrm{M}+\mathrm{H})^{+}$.

Ynamide 51 (35.0 $\mathrm{mg}, 42 \%$ yield).

$\mathrm{R}_{\mathrm{f}}=0.20$ (33\% EtOAc in Hexanes); clear oil; ${ }^{1} \mathrm{H}$ NMR $\left(300 \mathrm{MHz}, \mathrm{CDCl}_{3}\right) \delta 6.29(\mathrm{dt}, 1 \mathrm{H}, J=1.2,6.6 \mathrm{~Hz})$, $6.68(\mathrm{~d}, 1 \mathrm{H}, J=9.0 \mathrm{~Hz}), 7.09(\mathrm{~s}, 1 \mathrm{H}), 7.12(\mathrm{dd}, 1 \mathrm{H}, J=1.5,6.6 \mathrm{~Hz}), 7.27-7.41(\mathrm{~m}, 5 \mathrm{H}), 7.45$ (ddd, $J=2.1$, 6.6, 9.0 Hz); ${ }^{13} \mathrm{C}$ NMR $\left(75 \mathrm{MHz}, \mathrm{CDCl}_{3}\right) \delta 106.6,107.3,122.5,125.6,129.2,129.6,134.2,137.6,140.6,144.2$, 191.0; (thin film) $\mathrm{cm}^{-1} 3074(\mathrm{~m}), 1665$ (s), 1591 (s), 1532 (s), 1276 (m), 1144 (m); m/e (\% relative intensity) 276 and $277(100)(\mathrm{M}+\mathrm{H})^{+}$. 


\section{General Procedure for the Synthesis of Alkynyl Bromides.}

To a stirred solution of alkyne (1.0 equiv) in THF at $-78{ }^{\circ} \mathrm{C}$ was added dropwise $2.4 \mathrm{M} \mathrm{n}$-BuLi (1.2 equiv) solution in hexane. The resulting lithium acetylide solution was stirred for $30 \mathrm{~min}$, and $\mathrm{Br}_{2}$ (1.4 equiv) was added dropwise until red color persists. This light red solution was stirred at $-78{ }^{\circ} \mathrm{C}$ for an additional 15 min. The solution was quenched with saturated sodium thiosulfate solution, and subjected to standard aqueous work up using ether as the extract solvent. The organic extracts were combined, dried with sodium sulfate, and concentrated in vacuo. The residue was purified by silica gel column flash chromatography using hexane as isocratic eluent.

ACS Registry Numbers of Known Alkynyl Bromides.

Phenylbromoacetylene, 932-87-6;

1-bromo-1-octyne, 38761-67-0;

1-bromo-2-(triisopropylsilyl)ethyne, 111409-79-1;

(6-bromo-hex-5-ynyloxy)-t-butyl-dimethyl-silane, 124696-14-6;

(3-bromo-prop-2-ynyloxy)-t-butyl-diphenyl-silane, 397333-58-3;

1-bromo-2-(2-methoxyphenyl)acetylene, 199165-20-3.

\section{An Alternative Procedure Specifically for the Synthesis of 1-Bromo-2-(2-methoxynaphthnyl)acetylene.}

To a solution of triphenyl phosphine $(17.56 \mathrm{~g}, 67.0 \mathrm{mmol})$ and $\mathrm{CBr}_{4}(11.10 \mathrm{~g}, 33.41 \mathrm{mmol})$ in $\mathrm{CH}_{2} \mathrm{Cl}_{2}$ $(30 \mathrm{~mL})$ at $0^{\circ} \mathrm{C}$ were added dropwise via cannula a solution of 2-methoxylnaphthyl-1-aldehyde (4.79 g, 25.7 mmol) in $\mathrm{CH}_{2} \mathrm{Cl}_{2}(30 \mathrm{~mL})$ and $\mathrm{Et}_{3} \mathrm{~N}(3.37 \mathrm{~mL}, 25.7 \mathrm{mmol})$. The solution was stirred at $0^{\circ} \mathrm{C}$ for $1 \mathrm{~h}$. Hexanes $(400 \mathrm{~mL})$ were added to the solution and stirred for $30 \mathrm{~min}$ at $0^{\circ} \mathrm{C}$ and filtered cold through a bed of silica. The solution was concentrated under reduced pressure and purified by silica gel column chromatography (gradient: $0-50 \%$ EtOAc in hexanes) to afford dibromoalkene in $92 \%$ yield.

To a solution of the dibromoalkene prepared above (7.16 g, $20.9 \mathrm{mmol})$ and benzyltriethylammonium chloride $(4.19 \mathrm{~g}, 10.4 \mathrm{mmol})$ in $\mathrm{CH}_{2} \mathrm{Cl}_{2}(80 \mathrm{~mL})$ at $0^{\circ} \mathrm{C}$ was added a solution of $60 \% \mathrm{KOH}(54 \mathrm{~g})$ in $\mathrm{H}_{2} \mathrm{O}(40$ $\mathrm{mL}$ ). The solution was stirred for $1 \mathrm{~h}$ at $0^{\circ} \mathrm{C}$, extracted with $\mathrm{CH}_{2} \mathrm{Cl}_{2}$, dried over $\mathrm{Na}_{2} \mathrm{SO}_{4}$, and concentrated in vacuo. The desired alkynyl bromide product was isolated in $99 \%$ yield (5.45 g) and used without further purification.

$\mathrm{R}_{\mathrm{f}}=0.74$ (50\% EtOAc in hexane); yellow solid; ${ }^{1} \mathrm{H} \mathrm{NMR}\left(300 \mathrm{MHz}, \mathrm{CDCl}_{3}\right) \delta 4.03(\mathrm{~s}, 3 \mathrm{H}), 7.25(\mathrm{t}, 1 \mathrm{H}, J=$ $8.5 \mathrm{~Hz}), 7.38(\mathrm{dt}, 1 \mathrm{H}, J=1.0,8.0 \mathrm{~Hz}), 7.55(\mathrm{dt}, 1 \mathrm{H}, J=1.5,7.0 \mathrm{~Hz}), 7.78(\mathrm{~d}, 1 \mathrm{H}, J=8.5 \mathrm{~Hz}), 7.83(\mathrm{~d}, 1 \mathrm{H}, J$ $=8.5 \mathrm{~Hz}), 8.21(\mathrm{~d}, 1 \mathrm{H}, J=8.0 \mathrm{~Hz}) ;{ }^{13} \mathrm{C} \mathrm{NMR}\left(125 \mathrm{MHz}, \mathrm{CDCl}_{3}\right) \delta 55.8,57.9,75.0,112.7,124.5,125.2,127.8$, 130.7, 135.2; mass spectrum (EI): m/e (\% relative intensity) 260 (100) ( $\mathrm{M}^{+}$), 217 (95), 152 (25), 138 (50); m/e calcd for $\mathrm{C}_{13} \mathrm{H}_{9} \mathrm{BrNaO}, 282.9729$ measd. 282.9734. 